\title{
CIRED 2013: Bericht über Session 6 - Strommärkte und Regulierung
}

\section{H. Struber OVE}

Online publiziert am 11. Jänner 2014

(c) Springer Verlag Wien 2013

\section{Zusammenfassung}

In der Session 6 wurden in den Main- und Poster Sessions in Summe 80 Beiträge vorgestellt, 20 dieser Beiträge wurden in vier Themenblöcke unterteilt und den Konferenzteilnehmern in den Main Sessions vorgestellt. Die Session 6 war, schon bedingt durch die neuen Entwicklungen und aktuellen Themen wie z. B. Smart Metering, wie immer sehr gut besucht.

Die vier Themenblöcke wurden unter folgenden Titeln geführt:

- Auswirkung von Smart Meter und neuen Tarifen auf Kunden

- Regulierung und Marktentwicklung

- Zukünftige Herausforderungen und Aufgaben im Verteilernetzgeschäft

- Erfahrungen aus Smart Grids-Projekten

\section{Block 1 - Auswirkung von Smart Meter und neuen Tarifen} auf Kunden

Dieser Block und die dazugehörigen Beiträge waren sehr stark von den Smart Meter-Projekten und den Möglichkeiten von neuen Tarifmodellen geprägt. Diese Themen sind aufgrund der bereits durchgeführten Smart Meter-Roll-outs bzw. der sich gerade in Planung befindlichen Roll-outs in den verschiedenen europäischen Ländern erfahrungsgemäß bei allen Betroffenen und Beteiligten von großem Interesse.

Praktische Erfahrungsberichte wurden von Italien und Schweden vorgestellt, welche bereits seit einigen Jahren Erfahrungen mit der neuen Zählertechnologie gesammelt haben, bzw. auch aus Frankreich, wo aktuell Smart Meter implementiert werden. Aus Italien wurde des Weiteren auch von Erfahrungen mit Time of use-Tarifen berichtet. Die Möglichkeiten von Lastverschiebungen sind dabei zwar vorhanden, der Nutzen bzw. die Kundenersparnisse halten sich bei schwachen Preissignalen jedoch in Grenzen.

\section{Block 2 - Regulierung und Marktentwicklung}

Dieser Präsentationsblock war sehr stark von Beiträgen von Regulatoren, politischen Akteuren und Interessensverbänden geprägt, die einen interessanten und teilweise neuen Blickwinkel in die Session 6 brachten. Schwerpunkte bildeten dabei unter anderem die Berichte aus Finnland bezüglich Versorgungssicherheit aus Sicht der politischen Entscheidungsträger sowie aus Rumänien, wo es um die Abschaffung der regulierten Energietarife ging.

Dieser Block zeigte vor allem aber auch die Notwendigkeit auf, die Marktentwicklung weiter voranzutreiben und allen Akteuren einen Zugang zu den Märkten zu ermöglichen. Ein Beispiel, welchen Beitrag die Photovoltaik zum Regelenergiemarkt liefern kann, wurde unter anderem aus Deutschland vorgestellt.

\section{Block 3 - Zukünftige Herausforderungen und Aufgaben im Verteilernetzgeschäft}

Die zahlreichen Beiträge des dritten Blocks beschäftigten sich mit dem Thema Verteilernetzgeschäft, welches sich zukünftig ändern wird. Vorrangig ging es um die Implementierung eines aktiven Netzmanagements, das wesentlich weiter fortgeschritten sein soll, als die bisherigen Strategien zur Netzsteuerung dies ermöglichen. Einen wichtigen Aspekt bilden dabei auch die Auswirkungen der Laststeuerung im Verteilernetz, wo es jedoch noch wenig praktische Erfahrungen gibt.

Schließlich wurde, neben all den neuen Herausforderungen im Smart Grid, auch noch die klassische Frage behandelt, wie Asset Management im Verteilernetzgeschäft weiter optimiert werden kann. Im Speziellen wurden Vorschläge vorgestellt, wie Investitions- und Betriebskosten weiter optimiert werden können.

\section{Block 4 - Smart Grids-Projekte}

In vielen laufenden Smart Grids-Projekten werden derzeit ähnliche Herausforderungen und Gesamtkonzepte für eine Lösung untersucht. Vorgestellt wurden Projekte aus Italien, Spanien, Portugal und Schweden, welche Themen wie die aktive Einbindung der Kunden durch z. B. Smart Metering, Systemanforderungen bis hin zur Marktentwicklung behandelten. Besonderes Augenmerk galt dabei auch dem Projekt Ecogrid, das die wichtige Frage der Erarbeitung eines neuen Marktkonzeptes zum Ausgleich der fluktuierenden dezentralen Erzeugung mittels Lastflexibilisierung aufzeigte.

\section{Round Tables und Poster Tours}

In den vier Round Tables der Session 6 wurden unter anderem die Themen zukünftige Regulierung im Hinblick auf Investitionen und Marktentwicklung, Europäische Netzkodizes, Smart GridsErfahrungen und internationale Roadmaps, Kundenverhalten bis hin zu Smart Home behandelt bzw. diskutiert.

In den geführten Poster Tours hatten die Autoren wieder die Möglichkeit, ihre Beiträge einem interessierten Publikum zu präsentieren und direkt auf Fragen einzugehen bzw. in einen Dialog mit dem Fachpublikum zu treten. Die Poster Sessions waren, wie schon in den letzten Jahren, gut besucht und erwiesen sich aus Sicht des Autors wieder als echte Bereicherung der Konferenz.

Eine Auswahl von Beiträgen aus der Session 6 wird in den CIRED Info-Nachmittagen am 28. Jänner 2014 in Wien bzw. 4. Februar 2014 in Innsbruck vorgestellt. Sämtliche Beiträge der Main Session, der Round Tables und der Poster Session sind unter dem Link http://www.cired2013.org abrufbar.

22nd International Conference on Electricity Distribution, Stockholm, Schweden, 10. bis 13. Juni 2013.

Struber, Herwig, Salzburg Netz GmbH, Bayerhammerstraße 16, 5020 Salzburg Österreich (E-Mail: herwig.struber@salzburgnetz.at) 